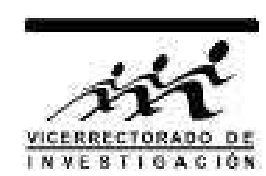

\title{
Estudio de la influencia de impurezas en la conductividad eléctrica de apatitas: el caso de CaHAp
}

\author{
R. M. Espinoza*y C. V. Landauro \\ Facultad de Ciencias Físicas, Universidad Nacional Mayor de San Marcos, AP 14-0149, Lima 14, Perú
}

Recibido 17 diciembre 2012 - Aceptado 15 febrero 2013

\begin{abstract}
En el presente trabajo se estudia la dependencia de la conductividad eléctrica de las apatitas respecto a la temperatura. Para ello, se propone un modelo de conductividad espectral que toma en cuenta las características más relevantes del material mencionado. La conductividad eléctrica calculada a partir de este modelo está en buen acuerdo con las medidas experimentales obtenidas en estos sistemas por Laghzizil et. al 1]. Adicionalmente, se observa un aumento de la conductividad eléctrica, en el rango de 1000 a $1300^{\circ} \mathrm{C}$, al incluir impurezas en el sistema.

Palabras claves: Apatitas, conductividad espectral, conductividad eléctrica.

\section{Study of the impurities influence on the electrical conductivity of apatites: the CaHAp case}

In the present work we study the temperature dependence of the electrical conductivity of apatites. For this purpose, we propose a spectral conductivity model which takes into account the most relevant characteristics of the material analyzed. The electrical conductivity obtained from this model is in good agreement with experimental data measured by Laghzizil et. al [1. Additionally, we also observe an increment of the electrical conductivity, in the range from 1000 to $1300^{\circ} \mathrm{C}$ ) after including impurities in the system.

Keywords: Apatites, spectral conductivity, electrical conductivity.

En las últimas décadas el avance de la tecnología para el tratamiento de diferentes tipos de enfermedades y dolencias ha incentivado a la comunidad científica hacia la búsqueda de nuevos materiales que puedan reemplazar o mejorar el funcionamiento de algún tejido u órgano dañado del cuerpo humano. A este nuevo tipo de materiales se les conoce como biomateriales.

Particularmente, los biomateriales relacionados a la reparación del tejido óseo son conocidos como biocerámicos dentro de los cuales hay un interés especial en los denominados fosfatos de calcio como la hidroxiapatita HAp -del inglés Hydroxyapatite-, $\mathrm{Ca}_{10}\left(\mathrm{PO}_{4}\right)_{6}(\mathrm{OH})_{2}, \mathrm{y}$ el fosfato tricálcico TCP -del inglés Tri-Calcium Phosphate-, $\mathrm{Ca}_{3}\left(\mathrm{PO}_{4}\right)_{2}$, que difieren uno del otro tanto en su composición química así como en sus propiedades físicas y químicas 2. La hidroxiapatita tiene una composición química muy similar a la del hueso humano, lo cual la hace biocompatible con los tejidos vivos. Esta biocompatibilidad posibilita su uso en variadas aplicaciones médicas, por ejemplo, como un posible material de implante óseo [2].

Mientras que un gran número de investigadores se han concentrado en el estudio de las propiedades mecánicas y la biocompatibilidad [3, 4] de estos materiales, otros investigadores se han dedicado al estudio de sus propiedades eléctricas [5] 8 . El estudio de estas propiedades en la HAp es muy variado. Por ejemplo, Fanovich y colaboradores [5] han usado las medidas eléctricas como una herramienta de caracterización para estudiar la evolución de microestructuras en la HAp 5. Por otro lado, Valdes y colaboradores 9 han examinado las propiedades dieléctricas de la HAp para entender su descomposición en el TCP como un resultado de la deshidratación de los iones $\mathrm{OH}^{-}$a temperaturas elevadas. Así, el concepto de estimulación eléctrica del implante, propuesta por Zakharov y colaboradores [10], es relevante para examinar la dependencia térmica de la permitividad y la pérdida dieléctrica de la HAp a temperaturas entre 20 y $500^{\circ} \mathrm{C}$. Como se puede apreciar, hay múltiples aplicaciones de las propiedades eléctricas

*respinozab1@correo.unmsm.edu.pe 
de la HAp pero no se ha especificado como es la conductividad eléctrica de la misma. En este sentido, en el presente estudio se analiza teóricamente la conductividad eléctrica de la HAp y el efecto de las impurezas sobre la misma.

El artículo está organizado como sigue: en la siguiente sección introducimos las relaciones básicas que usaremos en nuestros cálculos. Además, se indica el modelo empleado para la conductividad espectral. En la siguiente sección se presentan los resultados. Finalmente, las conclusiones son expuestas en la última sección.

\section{Dependencia con la temperatura de la conductividad eléctrica}

Para determinar la dependencia de la conductividad eléctrica con respecto a la temperatura empleamos la formulación de Kubo-Greenwood (KG) 11], dado por

$$
\sigma(T)=\int d \varepsilon \hat{\sigma}(\varepsilon)\left\{-\frac{\partial f(\varepsilon, \mu, T)}{\partial \varepsilon}\right\}
$$

donde $f(\varepsilon, \mu, T)$ es la función de distribución de FermiDirac, $\widehat{\sigma}(\varepsilon)$ es la conductividad espectral y $\mu$ es el potencial químico definido por

$$
\mu(T) \approx \varepsilon_{F}-\left(k_{B} T\right)^{2} \frac{\pi^{2}}{6}\left[\frac{\eta^{\prime}(\varepsilon)}{\eta(\varepsilon)}\right]_{\varepsilon_{F}}=\varepsilon_{F}-\xi T^{2},
$$

donde $\eta(\varepsilon)$ es la densidad de estados electrónicos (DOS) y $\eta^{\prime}(\varepsilon)$ es su derivada con respecto a la energía $\varepsilon$.

Para determinar la conductividad eléctrica de las apatitas es necesario conocer un modelo para $\widehat{\sigma}(\varepsilon)$, el cual debe contener las características más relevantes de este material.

\section{Modelando la conductividad espectral}

Enderby y Barnes 13 proponen un modelo para la conductividad espectral $\widehat{\sigma}(\varepsilon)$ de semiconductores líquidos a altas temperaturas de la forma,

$$
\widehat{\sigma}(\varepsilon)=\left\{\begin{array}{cc}
\alpha_{\nu}\left(\varepsilon_{\nu}-\varepsilon\right), & \varepsilon \leq \varepsilon_{\nu} \\
0 \quad, & \varepsilon_{\nu} \leq \varepsilon \leq \varepsilon_{c} \\
\alpha_{c}\left(\varepsilon-\varepsilon_{c}\right), & \varepsilon_{c} \leq \varepsilon
\end{array}\right.
$$

donde $\alpha_{\nu}, \alpha_{c}$ son parámetros libres de ajuste, $\varepsilon_{\nu}$ y $\varepsilon_{c}$ son los bordes de la banda de valencia y de conducción, respectivamente. El gap de energía, medido por $\Delta \varepsilon=\varepsilon_{c}-\varepsilon_{\nu}$, toma normalmente valores entre 0 y 500 $\mathrm{meV}$ [13. Un esbozo de éste modelo se muestra en la Fig.1.a.
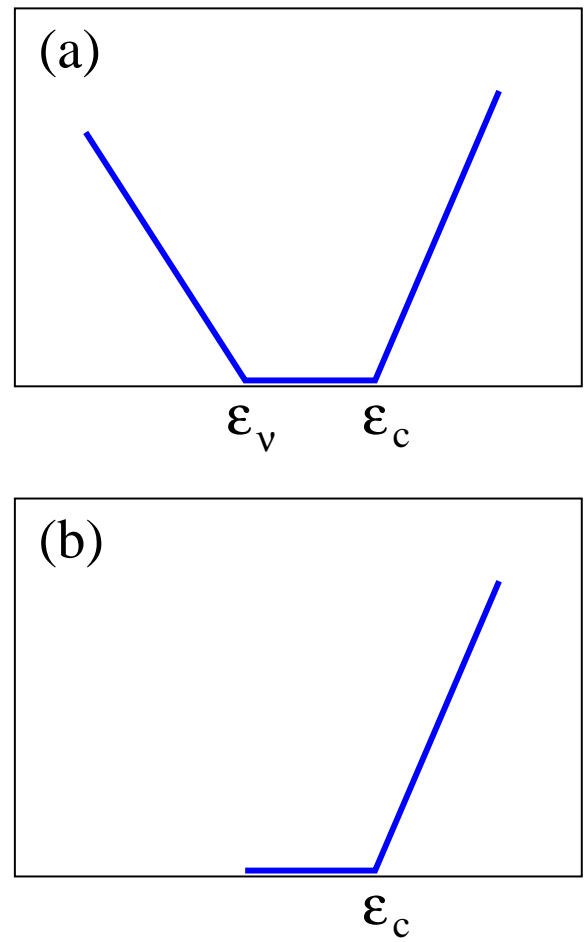

Figura 1: Representación esquemática para los modelos de conductividad espectral. (a) $\widehat{\sigma}(\varepsilon)$ dado por la Ec. (3) y (b) $\widehat{\sigma}(\varepsilon)$ dado por la Ec. (4).

Ahora, basándonos en el estudio bajo primeros principios realizado por Matsunaga y Kuwabara [14], en el cual se investiga la estructura electrónica de la hidroxiapatita, nosotros empleamos un modelo fenomenológico, muy similar al dado por la ecuación (3), para la conductividad espectral de las apatitas. Como el gap de energía en el caso de la HAp es grande [14] $(\Delta \varepsilon=6$ $\mathrm{eV}$ ), con respecto al rango entre 0 y $500 \mathrm{meV}$, se puede despreciar una pendiente. Por consiguiente, la ec. (3) se convierte en

$$
\widehat{\sigma}(\varepsilon)=\left\{\begin{array}{cc}
0 & \varepsilon \leq \varepsilon_{c} \\
\alpha_{c}\left(\varepsilon-\varepsilon_{c}\right), & \varepsilon_{c} \leq \varepsilon
\end{array}\right.
$$

Este modelo fenomenológico es usado como la conductividad espectral de las apatitas. Un esbozo de éste modelo se muestra en la Fig.1.b.

\section{Resultados}

Al reemplazar la Ec.(4) en la Ec.(11), consideramos que $\mu \approx \varepsilon_{F}$ y recordando que se cumple

$$
-\frac{\partial f(\varepsilon, \mu, T)}{\partial \varepsilon}=\frac{\beta}{4} \operatorname{sech}^{2}\left\{\frac{\beta}{2}(\varepsilon-\mu)\right\},
$$

obtenemos

$$
\sigma(T)=\int_{\varepsilon_{c}}^{\infty} d \varepsilon\left[\alpha_{c}\left(\varepsilon-\varepsilon_{c}\right)\right] \frac{\beta}{4} \operatorname{sech}^{2}\left\{\frac{\beta}{2}\left(\varepsilon-\varepsilon_{F}\right)\right\},
$$


con $\beta=\left(k_{B} T\right)^{-1}$ e integrando analíticamente la Ec. (5) obtenemos que

$$
\sigma(T)=\alpha_{c} k_{B} T \ln \left[1+\exp \left(-A_{c}\right)\right]
$$

donde

$$
A_{c}=\frac{\varepsilon_{c}-\varepsilon_{F}}{k_{B} T}
$$

De la Ec. (6) tenemos que para valores pequeños de $A_{c}$; es decir para $\left(\varepsilon_{c}-\varepsilon_{F}\right) \ll k_{B} T$, la dependencia de la conductividad eléctrica con la temperatura es prácticamente lineal

$$
\sigma(T) \approx \ln (2) \alpha_{c} k_{B} T, \quad \text { tal que } \quad\left(\varepsilon_{c}-\varepsilon_{F}\right) \ll k_{B} T,
$$

mientras que para valores grandes de $A_{c}$; es decir para $\left(\varepsilon_{c}-\varepsilon_{F}\right) \gg k_{B} T$, la conductividad eléctrica prácticamente se anula, esto es

$$
\sigma(T) \rightarrow 0, \text { tal que }\left(\varepsilon_{c}-\varepsilon_{F}\right) \gg k_{B} T .
$$

En general, tenemos que la dependencia de la conductividad eléctrica con la temperatura está dada por la Ec.(6). En la Fig.2 se representa gráficamente dicha dependencia. Los valores de los parámetros se han tomado tal que se ajusten a los valores experimentales (tomados de la Ref. 1]) para la HAp con Calcio (CaHAp). Dichos valores están dados por $\varepsilon_{c}=6.0 \mathrm{eV}, \varepsilon_{F}=4.85$ $\mathrm{eV}$ y $\alpha_{c}=1.45(\Omega-\mathrm{cm}-\mathrm{eV})^{-1}$.

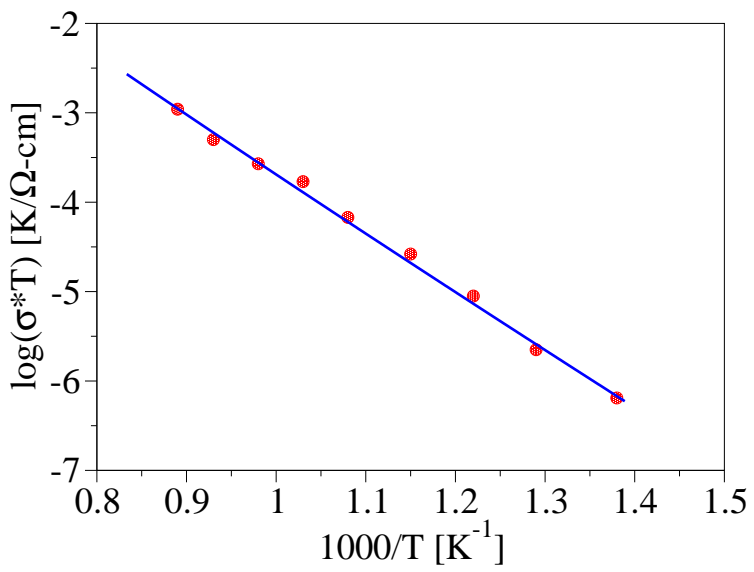

Figura 2: Se muestra la conductividad eléctrica como función de la temperatura, Ec. (6). Los valores de los parámetros se han tomado tal que se ajusten a los valores experimentales (tomados de la Ref. 1]) para la HAp con Calcio (CaHAp).

\section{Efecto de impurezas en la conductividad eléctrica de CaHAp}

Puesto que las impurezas son responsables de la aparición de estados localizados en las zonas del gap de la densidad de estados electrónicos, se propone un modelo del tipo de una gaussiana para considerar la contribución a la conductividad espectral debido a las impurezas. El ancho y la altura de la gaussiana dependen del porcentaje y el tipo de impurezas presentes en la HAp (o CaHAp). En nuestro caso el ancho y la altura se consideran como parámetros libres para ver su efecto en la dependencia de la conductividad eléctrica respecto a la temperatura. Dicho modelo se adiciona al modelo para la HAp sin impurezas, Ec.(4), tal que la conductividad espectral, considerando el efecto de las impurezas en la HAp, está dada por

$$
\widehat{\sigma}(\varepsilon)=\left\{\begin{array}{cc}
C \exp \left[-\frac{\left(\varepsilon-\varepsilon^{\prime}\right)^{2}}{2 \gamma^{2}}\right] & \left|\varepsilon_{c}-\varepsilon^{\prime}\right| \gg \gamma \\
\varepsilon_{c} \leq \varepsilon \\
\alpha_{c}\left(\varepsilon-\varepsilon_{c}\right) & \varepsilon_{c} \leq \varepsilon
\end{array}\right.
$$

donde $C, \varepsilon^{\prime}$ y $\gamma$ son parámetros libres de ajuste. Así, al reemplazar la Ec.(10) en la Ec1 tenemos que

$$
\sigma(T)=\sigma_{1}(T)+\sigma_{2}(T)
$$

donde

$$
\begin{array}{r}
\sigma_{1}(T)=\int_{-\infty}^{\varepsilon_{c}} d \varepsilon\left\{C \exp \left[-\frac{\left(\varepsilon-\varepsilon^{\prime}\right)^{2}}{2 \gamma^{2}}\right]\right\} \frac{\beta}{4} \times \\
\operatorname{sech}^{2}\left\{\frac{\beta}{2}\left(\varepsilon-\varepsilon_{F}\right)\right\}
\end{array}
$$

y

$$
\begin{aligned}
& \sigma_{2}(T)=\int_{\varepsilon_{c}}^{\infty} d \varepsilon\left[\alpha_{c}\left(\varepsilon-\varepsilon_{c}\right)\right] \frac{\beta}{4} \times \\
& \operatorname{sech}^{2}\left\{\frac{\beta}{2}\left(\varepsilon-\varepsilon_{F}\right)\right\},
\end{aligned}
$$

$\operatorname{con} \mu \approx \varepsilon_{F}$.

Para obtener una expresión analítica para $\sigma(T)$ solo nos falta determinar $\sigma_{1}(T)$ puesto que ya se tiene una expresión para $\sigma_{2}(T)$ (véase la Ec.([6]). Así, para determinar $\sigma_{1}(T)$ tenemos en cuenta que la función gaussiana se aproxima a cero cuando $\varepsilon \rightarrow \pm \infty$. Entonces, teniendo en cuenta lo anterior se puede cambiar el límite de integración superior $\varepsilon_{c}$ por $\infty$, con lo que obtenemos,

$$
\begin{aligned}
\sigma_{1}(T)=\frac{\beta C}{4} \int_{-\infty}^{\infty} d \varepsilon \exp [ & \left.-\frac{\left(\varepsilon-\varepsilon^{\prime}\right)^{2}}{2 \gamma^{2}}\right] \times \\
& \operatorname{sech}^{2}\left\{\frac{\beta}{2}\left(\varepsilon-\varepsilon_{F}\right)\right\}
\end{aligned}
$$

La relación

$$
\operatorname{sech}^{2}\left[\frac{\beta}{2}\left(\varepsilon-\varepsilon_{F}\right)\right]=\frac{d}{d \varepsilon}\left\{\frac{2}{\beta} \tanh \left[\frac{\beta}{2}\left(\varepsilon-\varepsilon_{F}\right)\right]\right\}
$$


es usada para la integración por partes, de la que obtenemos

$$
\begin{array}{r}
\sigma_{1}(T)=\frac{C}{2 \gamma^{2}} \int_{-\infty}^{\infty}\left(\varepsilon-\varepsilon^{\prime}\right) \exp \left[-\frac{\left(\varepsilon-\varepsilon^{\prime}\right)^{2}}{2 \gamma^{2}}\right] \times \\
\tanh \left[\frac{\beta}{2}\left(\varepsilon-\varepsilon_{F}\right)\right] d \varepsilon .
\end{array}
$$

Tomando en cuenta la expansión en serie de Taylor del término tangente hiperbólico [15] se tiene que

$$
\begin{aligned}
& \tanh \left[\frac{\beta}{2}\left(\varepsilon-\varepsilon_{F}\right)\right]=\sum_{n=1}^{\infty} \frac{(-1)^{n-1} 2^{2 n}\left(2^{2 n}-1\right)}{(2 n) !} \times \\
& B_{n}\left[\frac{\beta}{2}\left(\varepsilon-\varepsilon_{F}\right)\right]^{2 n-1}, \quad \text { tal que }\left|\varepsilon-\varepsilon_{F}\right|<\pi k_{B} T
\end{aligned}
$$

donde $B_{n}$ son los números de Bernoulli $\left(B_{1}=1 / 6\right.$, $\left.B_{2}=1 / 30, B_{3}=1 / 42, \cdots\right)$. Entonces, reemplazando la Ec. (16) en la Ec. (15) e integrando término por término, obtenemos

$$
\begin{gathered}
\sigma_{1}(T)=C \sum_{n=1}^{\infty} \frac{(-1)^{n-1} 2^{2 n}\left(2^{2 n}-1\right)}{(2 n) !} B_{n}\left[\frac{\beta \gamma}{\sqrt{2}}\right]^{2 n-1} \times \\
\sum_{\substack{k \\
\text { (0 ó par) }}}^{2 n-1} \frac{(2 n-1) !}{k !(2 n-1-k) !} \Delta^{k} \Gamma\left(\frac{2 n-k+1}{2}\right), \quad(17)
\end{gathered}
$$

donde $\Gamma$ es la función gamma [15] y

$$
\Delta=\frac{\left(\varepsilon^{\prime}-\varepsilon_{F}\right)}{\sqrt{2} \gamma}
$$

Considerando sólo los tres primeros términos de la Ec.(17) se obtiene la expresión analítica

$$
\begin{aligned}
\sigma_{1}(T) \approx \frac{C \sqrt{\pi}}{2 \sqrt{2}} & \left\{\frac{\gamma}{k_{B} T}-\left(\frac{\Delta^{2}+1 / 2}{2}\right) \frac{\gamma^{3}}{\left(k_{B} T\right)^{3}}+\right. \\
& \left.\left(\frac{\Delta^{4}+3 \Delta^{2}+3 / 4}{6}\right) \frac{\gamma^{5}}{\left(k_{B} T\right)^{5}}\right\} .
\end{aligned}
$$

Por lo tanto, de la Ec.(11) tenemos que la expresión analítica para $\sigma(T)$, con $\sigma_{1}(T)$ y $\sigma_{2}(T)$ dados por las ecs. (19) y (6), respectivamente, está dada por

$$
\begin{aligned}
& \sigma(T)= \frac{C \sqrt{\pi}}{2 \sqrt{2}}\left\{\frac{\gamma}{k_{B} T}-\left(\frac{\Delta^{2}+1 / 2}{2}\right) \frac{\gamma^{3}}{\left(k_{B} T\right)^{3}}+\right. \\
&\left.\left(\frac{\Delta^{4}+3 \Delta^{2}+3 / 4}{6}\right) \frac{\gamma^{5}}{\left(k_{B} T\right)^{5}}\right\}+ \\
& \alpha_{c} k_{B} T \ln \left[1+\exp \left(-A_{c}\right)\right],
\end{aligned}
$$

con

$$
\begin{aligned}
& \Delta=\frac{\left(\varepsilon^{\prime}-\varepsilon_{F}\right)}{\sqrt{2} \gamma}, \quad A_{c}=\frac{\varepsilon_{c}-\varepsilon_{F}}{k_{B} T}, \\
& \text { y con la condición }\left|\varepsilon_{c}-\varepsilon_{F}\right| \gg \gamma .
\end{aligned}
$$

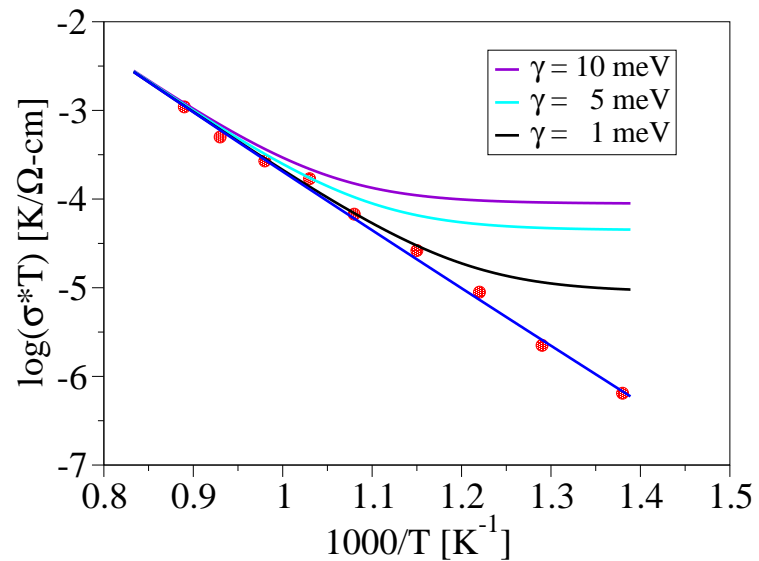

Figura 3: Se muestra la conductividad eléctrica como función de la temperatura, Ec.(20), para tres valores de $\gamma$, los cuales se muestran en la parte interna de la figura. Además, estas curvas se comparan con la conductividad eléctrica, línea azul, sin considerar los efectos de las impurezas, Ec. (6). Los demás valores para los parámetros se muestran en la Tabla 1.

En la Fig. 3 se muestra $\sigma(T)$, Ec.(20), tanto para el caso sin como con impurezas (línea azul). Los valores de los parámetros se tomaron tal que se ajusten a los valores experimentales, tomados de la Ref. [1, para CaHAp. Dichos valores están dados en la Tabla 1.

Como se puede apreciar en la Fig. 3, cuando $\gamma$ disminuye las curvas cada vez más se aproximan a la curva sin impurezas, línea de color azul, como es esperado, véase la Ec.(6).

En resumen, se puede notar que el modelo propuesto en el presente trabajo permite no solo modelar la dependencia de la conductividad eléctrica con la temperatura sino que también permite estudiar el efecto de las impurezas en la conductividad.

\begin{tabular}{ccccc}
\hline $\begin{array}{c}\alpha_{c} \\
(\Omega-\mathrm{cm}-\mathrm{eV})^{-1}\end{array}$ & $\begin{array}{c}\varepsilon_{c} \\
\mathrm{eV}\end{array}$ & $\begin{array}{c}\varepsilon_{F} \\
\mathrm{eV}\end{array}$ & $\begin{array}{c}C \\
(\Omega-\mathrm{cm})^{-1}\end{array}$ & $\begin{array}{c}\varepsilon^{\prime} \\
\mathrm{eV}\end{array}$ \\
\hline 1.45 & 6.0 & 4.85 & $1.24 \times 10^{-6}$ & 4.84 \\
\hline
\end{tabular}

Tabla 1: Valores de los parámetros para el modelo de $\sigma(T)$ dado por la Ec. (20).

\section{Conclusiones}

Para el estudio de la conductividad eléctrica de CaHAp se ha propuesto un modelo para la conductividad espectral, el cual está basado en cálculos $a b$ initio y que permite determinar la dependencia de la conductividad eléctrica con la temperatura en el rango de 1000 a $1300^{\circ} \mathrm{C}$. Los resultados obtenidos están en buen acuerdo con los datos experimentales reportados 
en la literatura. Más aún, se ha estudiado el efecto de las impurezas sobre la conductividad eléctrica. Dichas impurezas producen un aumento de la conductividad eléctrica, especialmente en el rango de 1000 a $1300^{\circ} \mathrm{C}$. Dicho incremento depende del tipo y porcentaje de las impurezas presentes en el material.

\section{Agradecimientos}

R. M. Espinoza agradece a la "Asociación Familia Zúñiga y Rivero" por la beca otorgada para la culminación de la redacción de la tesis de maestría y que ha permitido desarrollar, también, el presente trabajo.

\section{Referencias}

[1] A. Laghzizil, N. El Herch, A. Bouhaouss, G. Lorente y J. Macquete; J. Solid State Chemistry 156, 57 (2001).

[2] K. De Groot; Ceramics of calcium phosphates, Boca Raton: CRC Press, pags. 100-14 (1993).

[3] I. Sopyan, M. Mel, S. Ramesh y K. A. Khalid; Sci. Technol. Adv. Mater. 8, 116 (2007).

[4] K. A. Hing, S. M. Best, K. E. Tanner, W. Bonfield y P. A. Revell; J. Biomed. Mater. Res. A 68, 187 (2003).

[5] M. A. Fanovich, M. S. Castro y J. M. Porto Lopez, Ceram. Int. 25, 517 (1999).

[6] M. Sh. Kalil, H. H. Beheri y W. I. A. Fattah, Ceram. Int. 28, 451 (2002).

[7] C. C. Silva, M. P. F. Graca, M. A. Valente y A. S. B. Sombra, J. Non-Cryst. Sol. 352, 1490 (2006).
[8] J. P. Gittings, C. R. Bowen, A. C. E. Dent, I. G. Turner, F. R. Baxter y J. B. Chaudhuri, Acta Biomat. 5, 743 (2009).

[9] J. J. Prieto Valdes, A. Victorero Rodriguez y J. Guevara Carrio, J. Mater. Res. 10, 2174 (1995).

[10] N. A. Zakharov y V. P Orlovskii, Tech. Phys. Lett. 27, 629 (2001).

[11] R. Kubo, J. Phys. Soc. Jpn. 12, 570 (1957); D. A. Greenwood, Proc. Phys. Soc. London 71, 585 (1958).

[12] N. W. Ashcroft y N. D. Mermin, Solid State Physics, Saunders College, New York (1976).

[13] J. E. Enderby y A. C. Barnes, Rep. Progr. Phys. 53, 85 (1990).

[14] K. Matsunaga y A. Kuwabara, Phys. Rev. B 75, 014102 (2007).

[15] M. R. Spiegel, Manual de Fórmulas y Tablas Matemáticas, McGraw-Hill, México (1970). 